\title{
Preface
}

\section{Medications and Sleep}

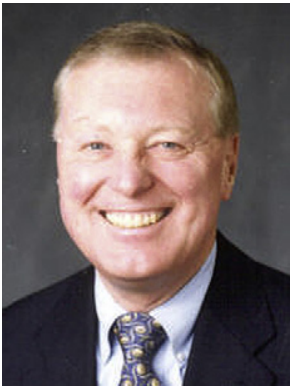

Timothy Roehrs, PhD

Guest Editor

Welcome to this issue of Sleep Medicine Clinics on the topic of Medications and Sleep. Any medication that crosses the blood-brain barrier carries the potential to alter sleep. Effects on sleep, either disruptive or therapeutic, may include alterations in the speed of sleep onset, the continuity of sleep, or the duration of sleep. Effects on sleep stages, often referred to as sleep architecture, may include changes in the normal age-related amounts of various sleep stages, or the ultradian rhythm of sleep, that is, the 90- to 120-min cycling through non-REM and REM sleep. Drug-induced changes in sleep and/or sleep stage architecture do not necessarily imply a disturbance of sleep. In some cases, the changes may be therapeutic, while in other cases, they may be benign or, at the least, not well understood. As clearly indicated in the first article by Watson et al, the complexity and range of neurobiologic mechanisms identified as controlling or influencing sleep and wake are the basis on which the many drugs discussed in this issue, with varying therapeutic targets and biological mechanisms, can affect sleep and wake.

The articles of the first half of this issue focus on the pharmacology of drug classes, their effects on sleep wake processes and theoretical discussions regarding how to assess hypnotic efficacy and safety, and the functional significance of sleep stage alterations. In Part 2, pharmacologic therapeutics for the major sleep disorders are described. These articles detail the major features of the various sleep disorders and then present various drugs used to treat the disorders. It is our hope that this issue will be informative and useful for both the relatively new sleep disorders practitioner and the more experienced practitioner, as well as for the clinical researcher.

Timothy Roehrs, PhD Department of Internal Medicine Sleep Disorders and Research Center Henry Ford Hospital Henry Ford Health System 2799 West Grand Boulevard, CFP-3

Detroit, MI 48202, USA

Department of Psychiatry and Behavioral Neuroscience Wayne State University, School of Medicine 2751 East Jefferson Detroit, MI 48207, USA

E-mail address: Troehrs1@hfhs.org 